\title{
Designing filters for polyphase filter banks
}

\author{
J.-H. Lee \\ W.-J. Kang
}

Indexing terms: Filter design, Polyphase filter banks

Abstract: The paper concerns the filter design problem for a recently-proposed polyphase filter bank with an arbitrary number of subband channels. We develop an analytical formula for the design of a prototype filter used in the polyphase filter bank. Compared with direct numerical design methods, this formula-based method allows the design of the required FIR lowpass prototype filter with a much lower complexity of operation and better filter bank performance. We further extend the theoretical results to two dimensions. Examples are also presented.

\section{Introduction}

Polyphase filter banks (PFB) based on quadrature mirror filters (QMF) have been successfully used to constitute a multirate signal processing system for splitting a signal into $N$ subbands and allowing the resynthesis of the signal from the subbands. Main applications of PFBs are in subband codng of speech signals [1, 2], TDM-FDM transmultiplexing systems $[3,4]$, and short-time spectral analysis $[5,6]$. Recently, the application of PFBs has been extended to two dimensions, for example for the subband coding of images [7,8], and 2-D short-time spectral analysis [9].

Considering the filter design for 1-D PFBs, many results have been reported in the literature [10-17]. Most of these methods employ numerical algorithms directly to minimise the associated error measure in the filter design process. Reference 18 describes an analytical formula to represent the error measure. As a result, the filter coefficients can be found by a straight nonlinear optimisation procedure, and a saving in computational load can be achieved. However, most of the above authors consider the design of QMFs. Moreover, methods for designing nonseparable 2-D prototype filters for use in 2-D PFBs were not available, although the 1-D methods $[17,18]$ were used for the design of a separable 2-D prototype filter for 2-D PFBs [9]. Recently, Reference 19 proposed an approach for the design of 2-D PFBs based on a constraint of cascaded Smith forms.

Based on nonsymmetrical frequency-band allocation in constructing bandpass filters for subband channels, Reference 20 describes a 1-D PFB with $\mathrm{N}$ arbitrary subbands. Each bandpass filter is a nonsymmetrical frequency-shifted version of a lowpass prototype filter. It is shown in Reference 20 that the new PFB possesses some advantages over conventional PFBs. A new 2-D

Paper 8470G (E3), received 20th May 1991

The authors are with the Department of Electrical Engineering, National Taiwan University, Taipei, Taiwan, Republic of China

IEE PROCEEDINGS-G, Vol. 139, No. 3, JUNE 1992
PFB with nonseparable filters is presented in Reference 21 because using nonseparable filters results in directional capability in the subband decomposition.

In this paper, we consider the filter design problem for the new PFBs. An analytical design formula for obtaining the coefficients of the required 1-D FIR lowpass prototype filter is derived in Section 2 . Compared with direct numerical design algorithms, the analytical design formula requires much less computational load and results in a smaller reconstruction error for the same number of subbands and filter length. In Section 3, we develop further an analytical formula for the design of a 2-D nonseparable FIR lowpass prototype filter for use in the new 2-D PFB, while in Section 4 we present simulation results for illustration.

\section{Analytical design formula for 1-D PFB}

The basic structure of a 1-D filter bank with $N$ subbands to be considered is shown in Fig. 1. Its efficient structure
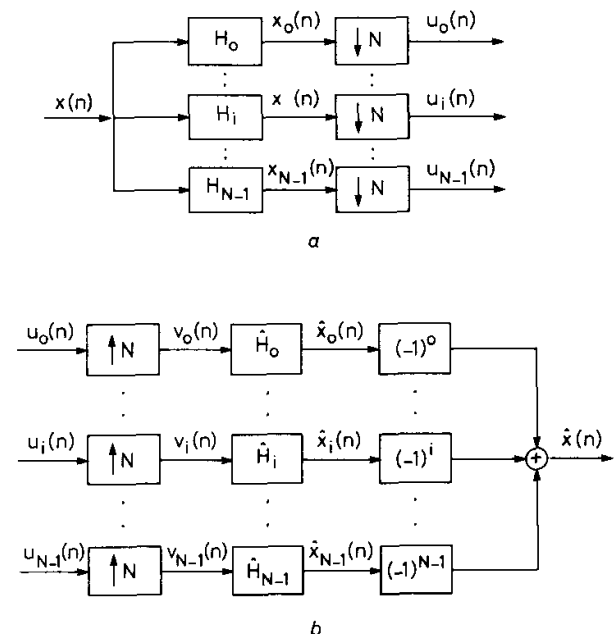

Fig. 1 I-D filter bank

a Analysis filter bank

$b$ Synthesis filter bank

based on a polyphase network and a fast Fourier transform (FFT) has been described in Reference 20. The bandpass filters $\hat{H}_{i}(\omega)$ and $H_{i}(\omega)$ are represented by

$$
H_{i}(\omega)=G_{i}(\omega)+G_{2 N-1-i}(\omega)
$$

and

$$
\hat{H}_{i}(\omega)=G_{i}(\omega)-G_{2 N-1-i}(\omega) \quad i=0,1,2, \ldots, N-1
$$


respectively, where $G_{i}(\omega) \triangleq G(\omega-i \pi / N)$ and $G(\omega)$ denotes the 1-D FIR lowpass prototype filter with length $M$. The associated conditions for perfect reconstruction are $[20]$

$$
\begin{aligned}
& |G(\omega)|=0 \quad|\omega| \geqslant \pi / N \\
& \sum_{k=0}^{2 N-1}|G(\omega-k \pi / N)|^{2}=N \quad 0 \leqslant \omega \leqslant 2 \pi \\
& M=N(2 L+1)+1
\end{aligned}
$$$$
\text { and }
$$

where $L$ is an integer. To design the FIR lowpass prototype filter $G(\omega)$ which approximates the condition of eqn. 3 in some optimum sense, we define an appropriate approximation error measure $E$ as

$$
E=E_{r}+\alpha E_{s}
$$

where $E_{r}$ denotes the related ripple energy over the whole frequency range and is given by

$$
E_{r}=\int_{0}^{\pi}\left\{\sum_{i=0}^{2 N-1}|G(\omega-l \pi / N)|^{2}-N\right\}^{2} d \omega
$$

$E_{s}$ denotes the stopband energy related to $G(\omega)$ and is given by

$$
E_{s}=\int_{\pi / N}^{\pi}|G(\omega)|^{2} d \omega
$$

$\alpha$ in eqn. 5 designates the relative weight between $E_{r}$ and $E_{s}$. To solve the problem of minimising $E$, some wellknown unconstrained numerical optimisation algorithms can be employed to find the filter coefficients of $G(\omega)$. However, at each iteration, we have to compute the Fourier transform of $G_{i}(\omega)$ for $i=0,1, \ldots, 2 N-1$, to obtain $E_{r}$ and $E_{s}$. This requires considerable computation time.

In the following, we derive a closed analytical formula to represent eqn. 5 and turn the minimisation problem into a direct search problem for the filter coefficients. Since $G(\omega)$ is in linear phase with length $M$, it can be expressed as

$$
\begin{aligned}
G(\omega)= & \exp (-j(M-1) \omega / 2) \\
& \times\left\{\sum_{n=0}^{M D} d(n) \cos ((n-(M-1) / 2) \omega)\right\}
\end{aligned}
$$

where $M D=(M-1) / 2, d((M-1) / 2)=g((M-1) / 2)$, and $d(n)=2 g(n)$ for $0 \leqslant n \leqslant(M-3) / 2$ if $M$ is odd, or $M D=M / 2-1, d(n)=2 g(n)$ for $0 \leqslant n \leqslant M / 2-1$ if $M$ is even. From eqn. 8 , we have

$$
\begin{aligned}
& \begin{aligned}
|G(\omega)|^{2}= & \sum_{m=0}^{M D} \sum_{n=0}^{M D} d(m) d(n) \cos ((m-(M-1) / 2) \omega) \\
& \times \cos ((n-(M-1) / 2) \omega) \\
= & \frac{1}{2} \sum_{m=0}^{M D} \sum_{n=0}^{M D} d(m) d(n)(\cos ((m-n) \omega) \\
& +\cos ((m+n-M+1) \omega))
\end{aligned} \\
& \text { and } \quad \\
& \qquad \begin{array}{l}
\sum_{k=0}^{2 N-1}|G(\omega-k \pi / N)|^{2} \\
=\frac{1}{2} \sum_{m=0}^{M D} \sum_{n=0}^{M D} d(m) d(n) \sum_{k=0}^{2 N-1} \cos ((m-n)(\omega-k \pi / N) \\
\quad+\cos ((m+n-M+1)(\omega-k \pi / N))) \triangleq F(\omega) \quad(10)
\end{array}
\end{aligned}
$$

364
Next, based on the fact that

$$
\begin{aligned}
& \sum_{k=0}^{2 N-1} \cos (l(\omega-k \pi / N)) \\
& \quad= \begin{cases}2 N \cos (l \omega) & l / 2 N=\text { integer } \\
0 & \text { else }\end{cases}
\end{aligned}
$$

eqn. 10 can be rewritten as

where

$$
r(l)= \begin{cases}\cos (l \omega) & l / 2 N=\text { integer } \\ 0 & \text { else }\end{cases}
$$

Now consider the integral given in eqn. 6. Expanding the squared integrand, we obtain

$$
E_{r}=\int_{0}^{\pi}\left(N^{2}-2 N F(\omega)+F^{2}(\omega)\right) d \omega
$$

Substituting eqn. 11 into eqn. 13 and using the fact that

$$
\int_{0}^{\pi} \cos (k \omega) d \omega= \begin{cases}\pi & k=0 \\ 0 & \text { else }\end{cases}
$$

we can show that the integration of eqn. 13 gives

$$
\begin{aligned}
E_{r}= & N^{2} \pi\left(1-2\left(\sum_{n=0}^{M D} d^{2}(n)+d^{2}((M-1) / 2)\right)\right. \\
& \left.+\frac{1}{2} \sum_{i=0}^{M D} \sum_{j=0}^{M D} \sum_{m=0}^{M D} \sum_{n=0}^{M D} d(i) d(j) d(m) d(n)\right)
\end{aligned}
$$

In eqn. 15 , the term $d^{2}((M-1) / 2)$ exists only when $M$ is odd and the last sum term exists only when one of the following combinations of the four integers $i, j, m, n$ holds

(a) $m-n=2 N k_{1}$ and $i-j= \pm 2 N k_{1}$

(b) $m-n=2 N k_{2}$ and $i+j-M+1= \pm 2 N k_{2}$

(c) $m+n-M+1=2 N k_{3}$ and $i+j-\bar{M}+1= \pm 2 N k_{3}$

for $0 \leqslant i, j, m, n \leqslant M D$ and integers $k_{1}, k_{2}, k_{3}$.

Next, consider $E_{s}$ in eqn. 7 . Substituting eqn. 9 into eqn. 7 and allowing for the fact that

$$
\int_{\pi / N}^{\pi} \cos (k \omega) d \omega= \begin{cases}\pi(1-1 / N) & k=0 \\ (-\sin (k \pi / N)) / k & \text { else }\end{cases}
$$

integration yields

$$
\begin{gathered}
E_{s}=\frac{1}{2} \sum_{m=0}^{M D} \sum_{n=0}^{M D} d(m) d(n)(s(m-n) \\
+s(m+n-M+1))
\end{gathered}
$$

where

$$
s(l)=\left\{\begin{array}{cl}
\pi(1-1 / N) & l=0 \\
-(\sin (\pi / N)) / l & l \neq 0 \\
0 & \text { else }
\end{array} \text { and } l / N \neq\right. \text { integer }
$$

From eqns. 15 and 17 , we note that the approximation error measure $E$ of eqn. 5 can be expressed as a closed analytical formula in terms of the filter coefficients $g(0)$, $g(1), \ldots, g(M-1)$. Hence, the computation of the filter coefficients by minimising $E$ can be performed by utilising a simple direct search method.

IEE PROCEEDINGS-G, Vol. 139, No. 3, JUNE 1992 
In this section, we extend the above 1-D theoretical results to the 2-D case. Consider the 2-D filter bank shown in Fig. 2. The number of subband channels is $N_{1} N_{2}$, where $N_{1}$ and $N_{2}$ are two arbitrary integers. An efficient structure consisting of a 2-D polyphase network and a 2-D FFT for the 2-D filter bank has been described in Reference 22. The bandpass filters $H_{i, j}\left(\omega_{1}, \omega_{2}\right)$ and $\hat{H}_{i, j}\left(\omega_{1}, \omega_{2}\right)$ are represented by

$$
\begin{aligned}
H_{i, j}\left(\omega_{1}, \omega_{2}\right)= & G_{i, j}\left(\omega_{1}, \omega_{2}\right)+G_{i, 2 N_{2}-1-j}\left(\omega_{1}, \omega_{2}\right) \\
& +G_{2 N_{1}-1-i, j}\left(\omega_{1}, \omega_{2}\right) \\
& +G_{2 N_{1}-1-i, 2 N_{2}-1-j}\left(\omega_{1}, \omega_{2}\right)
\end{aligned}
$$
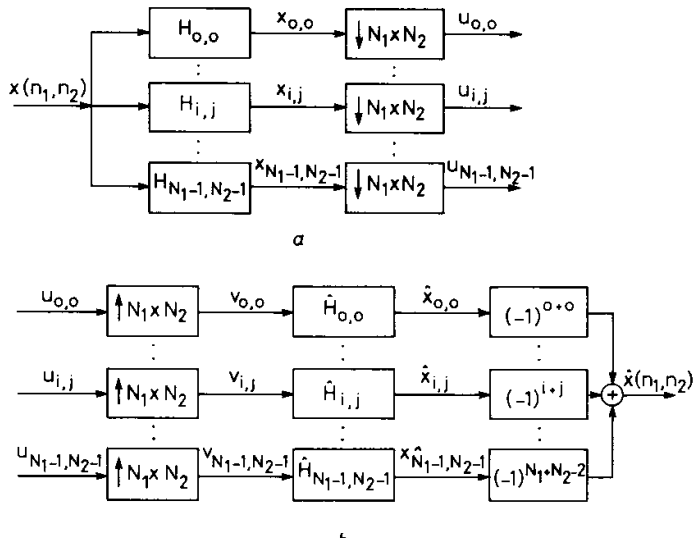

Fig. 2 2-D filter bank

a 2-D analysis filter bank

$b$ 2-D synthesis filter bank

and

$$
\begin{aligned}
\hat{H}_{i, j}\left(\omega_{1}, \omega_{2}\right)= & G_{i, j}\left(\omega_{1}, \omega_{2}\right)-G_{i, 2 N_{2}-1-j}\left(\omega_{1}, \omega_{2}\right) \\
& -G_{2 N_{1}-1-i, j}\left(\omega_{1}, \omega_{2}\right) \\
& +G_{2 N_{1}-1-i, 2 N_{2}-1-j}\left(\omega_{1}, \omega_{2}\right)
\end{aligned}
$$

where $G_{i, j}\left(\omega_{1}, \omega_{2}\right) \triangleq G\left(\omega_{1}-i \pi / N_{1}, \omega_{2}-j \pi / N_{2}\right), i=0,1$, $\ldots, N_{1}-1$ and $j=0,1, \ldots, N_{2}-1 . G\left(\omega_{1}, \omega_{2}\right)$ is a 2-D FIR lowpass prototype filter with length $M_{1} \times M_{2}$ and impulse response $g(m, n)$. Consider that $G\left(\omega_{1}, \omega_{2}\right)$ is nonseparable. The associated conditions for perfect reconstruction are

$$
\begin{aligned}
& \left|G\left(\omega_{1}, \omega_{2}\right)\right|=0 \quad\left|\omega_{1}\right| \geqslant \pi / N_{1} \text { and }\left|\omega_{2}\right| \geqslant \pi / N_{2} \quad(19) \\
& \sum_{k_{1}=0}^{2 N_{1}-1} \sum_{k_{2}=0}^{2 N_{2}-1}\left|G\left(\omega_{1}-k_{1} \pi / N_{1}, \omega_{2}-k_{2} \pi / N_{2}\right)\right|^{2}=N_{1} N_{2}
\end{aligned}
$$

$$
\begin{aligned}
& M_{1}=N_{1}\left(2 L_{1}+1\right)+1 \\
& M_{2}=N_{2}\left(2 L_{2}+1\right)+1
\end{aligned}
$$

and

$$
\begin{aligned}
G\left(\omega_{1}\right. & \left.-l_{1} \pi / N_{1}, \omega_{2}-l_{2} \pi / N_{2}\right) \\
& \times G\left(\omega_{1}-\left(l_{1}+1\right) \pi / N_{1}, \omega_{2}-\left(l_{2}+1\right) \pi / N_{2}\right) \\
= & G\left(\omega_{1}-\left(l_{1}+1\right) \pi / N_{1}, \omega_{2}-l_{2} \pi / N_{2}\right) \\
& \times G\left(\omega_{1}-l_{1} \pi / N_{1}, \omega_{2}-\left(l_{2}+1\right) \pi / N_{2}\right)
\end{aligned}
$$

where $L_{1}, L_{2}, l_{1}$, and $l_{2}$ are integers. Furthermore, with linear phase, $G\left(\omega_{1}, \omega_{2}\right)$ can be written as

$$
\begin{aligned}
G\left(\omega_{1}, \omega_{2}\right)= & \exp \left\{-j\left(\left(M_{1}-1\right) \omega_{1}+\left(M_{2}-1\right) \omega_{2}\right) / 2\right\} \\
& \times \sum_{m=0}^{M x} \sum_{n=0}^{M y} d(m, n) \cos \left(\left(m-\left(M_{1}-1\right) / 2\right) \omega_{1}\right) \\
& \times \cos \left(\left(n-\left(M_{2}-1\right) / 2\right) \omega_{2}\right)
\end{aligned}
$$

where $M x=\left(M_{1}-1\right) / 2$ if $M_{1}$ is odd and $M x=M_{1} / 2$ -1 if $M_{1}$ is even; $M y=\left(M_{2}-1\right) / 2$ if $M_{2}$ is odd and $M y=M_{2} / 2-1$ if $M_{2}$ is even; $d\left(m,\left(M_{2}-1\right) / 2\right)=2 g(m$, $\left.\left(M_{2}-1\right) / 2\right)$ if $M_{2}$ is odd and $d\left(\left(M_{1}-1\right) / 2, n\right)=2 g\left(\left(M_{1}\right.\right.$ $-1) / 2, n)$ if $M_{1}$ is odd; $d\left(\left(M_{1}-1\right) / 2,\left(M_{2}-1\right) / 2\right)=$ $g\left(\left(M_{1}-1\right) / 2,\left(M_{2}-1\right) / 2\right)$ if $M_{1}$ and $M_{2}$ are odd; and $d(m, n)=4 g(m, n)$ elsewhere.

Using eqn. 21 and performing some algebraic manipulations, we obtain

$\left|G\left(\omega_{1}, \omega_{2}\right)\right|^{2}$

$$
\begin{aligned}
= & \frac{1}{4} \sum_{m_{1}=0}^{M x} \sum_{n_{1}=0}^{M y} \sum_{m_{2}=0}^{M x} \sum_{n_{2}=0}^{M y} d\left(m_{1}, n_{1}\right) d\left(m_{2}, n_{2}\right) \\
& \times\left(\cos \left(\left(m_{1}-m_{2}\right) \omega_{1}\right)\right. \\
& \left.+\cos \left(\left(m_{1}+m_{2}-M_{1}+1\right) \omega_{1}\right)\right) \\
& \times\left(\cos \left(\left(n_{1}-n_{2}\right) \omega_{2}\right)\right. \\
& \left.+\cos \left(\left(n_{1}+n_{2}-M_{2}+1\right) \omega_{2}\right)\right)
\end{aligned}
$$

Further, from eqn. 24, we obtain

$$
\begin{aligned}
& \sum_{k_{1}=0}^{2 N_{1}-1} \sum_{k_{2}=0}^{2 N_{2}-1}\left|G\left(\omega_{1}-k_{1} \pi / N_{1}, \omega_{2}-k_{2} \pi / N_{2}\right)\right|^{2} \\
& =\frac{1}{4} \sum_{m_{1}=0}^{M x} \sum_{n_{1}=0}^{M y} \sum_{m_{2}=0}^{M x} \sum_{n_{2}=0}^{M y} d\left(m_{1}, n_{1}\right) d\left(m_{2}, n_{2}\right) \\
& \quad \times\left(\sum _ { k _ { 1 } = 0 } ^ { 2 N _ { 1 } - 1 } \left(\cos \left(\left(m_{1}-m_{2}\right)\left(\omega_{1}-k_{1} \pi / N_{1}\right)\right)\right.\right. \\
& \left.\left.\quad+\cos \left(\left(m_{1}+m_{2}-M_{1}+1\right)\left(\omega_{1}-k_{1} \pi / N_{1}\right)\right)\right)\right) \\
& \quad \times\left(\sum _ { k _ { 2 } = 0 } ^ { 2 N _ { 2 } - 1 } \left(\cos \left(\left(n_{1}-n_{2}\right)\left(\omega_{2}-k_{2} \pi / N_{2}\right)\right)\right.\right. \\
& \left.\left.\quad+\cos \left(\left(n_{1}+n_{2}-M_{2}+1\right)\left(\omega_{2}-k_{2} \pi / N_{2}\right)\right)\right)\right) \\
& \triangleq
\end{aligned}
$$

Based on eqn. 11, eqn. 25 can be rewritten as

$F\left(\omega_{1}, \omega_{2}\right)$

$$
\begin{aligned}
= & N_{1} N_{2} \sum_{m_{1}=0}^{M x} \sum_{n_{1}=0}^{M y} \sum_{m_{2}=0}^{M x} \sum_{n_{2}=0}^{M y} d\left(m_{1}, n_{1}\right) d\left(m_{2}, n_{2}\right) \\
& \times\left\{u\left(\omega_{1}, m_{1}-m_{2}\right)+u\left(\omega_{1}, m_{1}+m_{2}-M_{1}+1\right)\right\} \\
& \times\left\{u\left(\omega_{2}, n_{1}-n_{2}\right)+u\left(\omega_{2}, n_{1}+n_{2}-M_{2}+1\right)\right\}
\end{aligned}
$$

where

$$
u\left(\omega_{i}, k_{i}\right)= \begin{cases}\cos \left(k_{i} \omega_{i}\right) & k_{i} / 2 N_{i}=\text { integer } \\ 0 & \text { else }\end{cases}
$$

According to the proposed approximation error measure $E$ in eqn. $5, E_{r}$ and $E_{s}$ are given by

$$
E_{r}=\int_{0}^{\pi} \int_{0}^{\pi}\left(N_{1} N_{2}-F\left(\omega_{1}, \omega_{2}\right)\right)^{2} d \omega_{1} d \omega_{2}
$$


and

$$
\begin{aligned}
E_{s}= & \int_{\pi / N_{2}}^{\pi} \int_{0}^{\pi}\left|G\left(\omega_{1}, \omega_{2}\right)\right|^{2} d \omega_{1} d \omega_{2} \\
& +\int_{0}^{\pi / N_{2}} \int_{\pi / N_{1}}^{\pi}\left|G\left(\omega_{1}, \omega_{2}\right)\right|^{2} d \omega_{1} d \omega_{2}
\end{aligned}
$$

respectively for the 2-D case. Expanding the squared integrand of eqn. 27 yields

$$
\begin{aligned}
E_{r}= & \int_{0}^{\pi} \int_{0}^{\pi}\left(N_{1}^{2} N_{2}^{2}-2 N_{1} N_{2} F\left(\omega_{1}, \omega_{2}\right)\right. \\
& \left.+F^{2}\left(\omega_{1}, \omega_{2}\right)\right) d \omega_{1} d \omega_{2}
\end{aligned}
$$

Using eqns. 14 and 26 and performing algebraic manipulations, we obtain

$$
\begin{aligned}
E_{r}= & N_{1}^{2} N_{2}^{2} \pi^{2}-2 N_{1}^{2} N_{2}^{2} \pi^{2} \sum_{i=1}^{4} P_{i} \\
& +\left(N_{1}^{2} N_{2}^{2} \pi^{2} / 4\right) \sum d\left(m_{1}, n_{1}\right) d\left(m_{2}, n_{2}\right) \\
& \times d\left(i_{1}, j_{1}\right) d\left(i_{2}, j_{2}\right)
\end{aligned}
$$

where

$$
\begin{aligned}
& P_{1}=\sum_{m_{2}=0}^{M x} \sum_{n_{2}=0}^{M_{y}} d^{2}\left(m_{2}, n_{2}\right) \\
& P_{2}= \begin{cases}\sum_{m_{2}=0}^{M x} d^{2}\left(m_{2},\left(M_{2}-1\right) / 2\right) & M_{2}=\text { odd } \\
0 & \text { else }\end{cases} \\
& P_{3}= \begin{cases}\sum_{n_{2}=0}^{M y} d^{2}\left(\left(M_{1}-1\right) / 2, n_{2}\right) & M_{1}=\text { odd } \\
0 & \text { else }\end{cases} \\
& P_{4}= \begin{cases}d^{2}\left(\left(M_{1}-1\right) / 2,\left(M_{2}-1\right) / 2\right) \\
M_{1}=\text { odd } \text { and } M_{2}=\text { odd } \\
0 & \text { else }\end{cases}
\end{aligned}
$$

and the summation of the last term is taken over $0 \leqslant m_{1}$, $m_{2}, i_{1}, i_{2} \leqslant M x$ and $0 \leqslant n_{1}, n_{2}, j_{1}, j_{2} \leqslant M y$. Furthermore, this summation exists only when one of the following combinations of integer indexes holds.

(a) $\left\{\begin{array}{l}m_{1}-m_{2}=2 N_{1} k \text { and } i_{1}-i_{2}= \pm 2 N_{1} k \\ n_{1}-n_{2}=2 N_{2} l \text { and } j_{1}-j_{2}= \pm 2 N_{2} l\end{array}\right.$

(b) $\left\{m_{1}-m_{2}=2 N_{1} k\right.$ and $i_{1}-i_{2}= \pm 2 N_{1} k$

$\left\{\begin{array}{l}n_{1}-n_{2}=2 N_{2} l \text { and } j_{1}+j_{2}-M_{1}+1= \pm 2 N_{2} l \\ \text { la }\end{array}\right.$

(c) $\left\{m_{1}-m_{2}=2 N_{1} k\right.$ and $i_{1}+i_{2}-M_{1}+1= \pm 2 N_{1} k$

$\left\{n_{1}-n_{2}=2 N_{2} l\right.$ and $j_{1}-j_{2}= \pm 2 N_{2} l$

(d) $\left\{m_{1}-m_{2}=2 N_{1} k\right.$ and $i_{1}-i_{2}= \pm 2 N_{1} k$

$\left\{n_{1}+n_{2}-M_{2}+1=2 N_{2} l\right.$ and $j_{1}-j_{2}= \pm 2 N_{2} l$

$\left\{m_{1}-m_{2}=2 N_{1} k\right.$ and $i_{1}-i_{2}= \pm 2 N_{i} k$

(e) $\left\{n_{1}+n_{2}-M_{2}+1=2 N_{2} l\right.$ and $j_{1}+j_{2}-M_{2}+1= \pm 2 N_{2} l$

(f) $\left\{m_{1}+m_{2}-M_{1}+1=2 N_{1} k\right.$ and $i_{1}-i_{2}= \pm 2 N_{1} k$

(f) $\left\{n_{1}-n_{2}=2 N_{2} l\right.$ and $j_{1}-j_{2}= \pm 2 N_{2} l$

(g) $\left\{m_{1}-m_{2}=2 N_{1} k\right.$ and $i_{1}+i_{2}-M_{1}+1= \pm 2 N_{1} k$

$\left\{n_{1}-n_{2}=2 N_{2} l\right.$ and $j_{1}+j_{2}-M_{2}+1= \pm 2 N_{2} l$

(h) $\left\{m_{1}-m_{2}=2 N_{1} k\right.$ and $i_{1}+i_{2}-M_{1}+1= \pm 2 N_{1} k$

h) $\left\{n_{1}+n_{2}-M_{2}+1=2 N_{2} l\right.$ and $j_{1}-j_{2}= \pm 2 N_{2} l$

(i) $\left\{m_{1}+m_{2}-M_{1}+1=2 N_{1} k\right.$ and $i_{1}-i_{2}= \pm 2 N_{1} k$ i) $\left\{\begin{array}{l}n_{1}+n_{2}-M_{2}+1=2 N_{2} l \text { and } j_{1}-j_{2}= \pm 2 N_{2} l\end{array}\right.$ (j) $\left\{m_{1}+m_{2}-M_{1}+1=2 N_{1} k\right.$ and $i_{1}-i_{2}= \pm 2 N_{1} k$

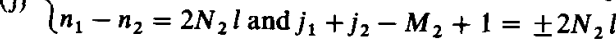

$\left\{\begin{array}{l}m_{1}-m_{2}=2 N_{1} k \text { and } i_{1}+i_{2}-M_{1}+1= \pm 2 N_{1} k \\ \text { (k) }\end{array}\right.$

(k) $\left\{n_{1}+n_{2}-M_{2}+1=2 N_{2} l\right.$ and $j_{1}+j_{2}-M_{2}+1= \pm 2 N_{2} l$

$\left\{m_{1}+m_{2}-M_{1}+1=2 N_{1} k\right.$ and $i_{1}-i_{2}= \pm 2 N_{1} k$

(l) $\left\{n_{1}+n_{2}-M_{2}+1=2 N_{2} l\right.$ and $j_{1}+j_{2}-M_{2}+1= \pm 2 N_{2} l$

(m) $\left\{\begin{array}{l}m_{1}+m_{2}-M_{1}+1=2 N_{1} k \\ \quad \text { and } i_{1}+i_{2}-M_{1}+1= \pm 2 N_{1} k \\ n_{1}-n_{2}=2 N_{2} l \text { and } j_{1}-j_{2}= \pm 2 N_{2}\end{array}\right.$

(n) $\left\{\begin{array}{l}m_{1}+m_{2}-M_{1}+1=2 N_{1} k \\ \text { and } i_{1}+i_{2}-M_{1}+1= \pm 2 N_{1} k\end{array}\right.$ $n_{1}-n_{2}=2 N_{2} l$ and $j_{1}+j_{2}-M_{2}+1= \pm 2 N_{2} l$

$\left\{m_{1}+m_{2}-M_{1}+1=2 N_{1} k\right.$

(o) $\left\{\right.$ and $i_{1}+i_{2}-M_{1}+1= \pm 2 N_{1} k$ $n_{1}+n_{2}-M_{2}+1=2 N_{2} l$ and $j_{1}-j_{2}= \pm 2 N_{2} l$

(p) $\left\{\begin{array}{l}m_{1}+m_{2}-M_{1}+1=2 N_{1} k \\ \quad \text { and } i_{1}+i_{2}-M_{1}+1= \pm 2 N_{1} k \\ n_{1}+n_{2}-M_{2}+1=2 N_{2} l \\ \text { and } j_{1}+j_{2}-M_{2}+1= \pm 2 N_{2} l\end{array}\right.$

where $k$ and $l$ are integers.

Next, substituting eqn. 24 into eqn. 28 and using

$\int_{0}^{\pi / N} \cos (k \omega) d \omega= \begin{cases}\pi / N & k=0 \\ (\sin (k \pi / N)) / k & \text { else }\end{cases}$

and performing algebraic manipulations yields

$$
\begin{aligned}
E_{s}= & \frac{1}{4} \sum d\left(m_{1}, n_{1}\right) d\left(m_{2}, n_{2}\right)\left\{\left(R_{2}\left(n_{1}-n_{2}\right)\right.\right. \\
& \left.+R_{2}\left(n_{1}+n_{2}-M_{2}+1\right)\right)\left(P\left(m_{1}-m_{2}\right)\right. \\
& \left.+P\left(m_{1}+m_{2}-M_{1}+1\right)\right)+\left(Q_{2}\left(n_{1}-n_{2}\right)\right. \\
& \left.+Q_{2}\left(n_{1}+n_{2}-M_{2}+1\right)\right)\left(R_{1}\left(m_{1}-m_{2}\right)\right. \\
& \left.\left.+R_{1}\left(m_{1}+m_{2}-M_{1}+1\right)\right)\right\}
\end{aligned}
$$

where the summation is taken over $0 \leqslant m_{1}, m_{2} \leqslant M x$ and $0 \leqslant n_{1}, n_{2} \leqslant M y$, and

$$
\begin{aligned}
& P(l)= \begin{cases}\pi & l=0 \\
0 & \text { else }\end{cases} \\
& Q_{i}(l)=\left\{\begin{array}{ll}
\pi / N_{i} & l=0 \\
\left(\sin \left(l \pi / N_{i}\right)\right) / l & l \neq 0 \\
0 & \text { else }
\end{array} \text { and } l / N_{i} \neq\right. \text { integer } \\
& R_{i}(l)=\left\{\begin{array}{cl}
\pi\left(1-1 / N_{i}\right) & l=0 \\
-\left(\sin \left(l \pi / N_{i}\right)\right) / l & l \neq 0 \\
0 & \text { else }
\end{array} \text { and } l / N_{i} \neq\right. \text { integer }
\end{aligned}
$$

for $i=1,2$. Hence, we note from eqns. 30 and 34 that the approximation error measure $E$ for the 2-D case can be expressed as a closed analytical formula in terms of the filter coefficients. Therefore, the filter coefficients $g(m, n)$ of $G\left(\omega_{1}, \omega_{2}\right)$ can also be found by utilising a simple direct search algorithm. In the following section, evaluation of computational complexity and simulation results are presented to confirm the above theoretical work. 


\section{Experimental examples}

4.1 1- $D$ design example

The filter length $M$ and the relative weight $\alpha$ were set to 16 and 0.1 , respectively. The number of subbands $N$ was three. Based on the analytical formulas presented in Section 2, we used a steepest-descent algorithm to iter-

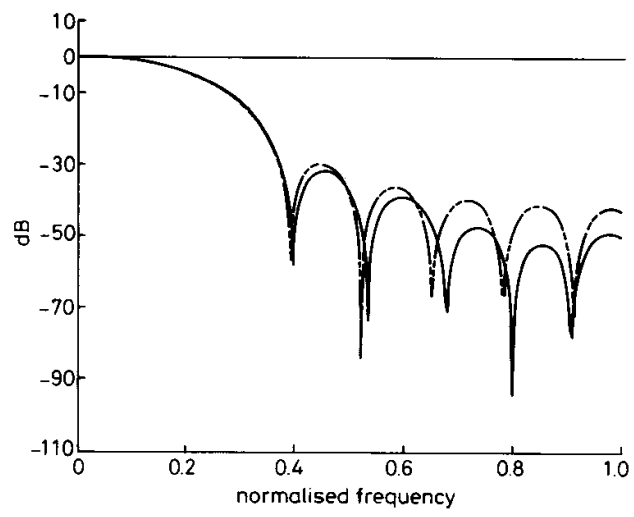

Fig. 3 Magnitude response of $G(\omega)$

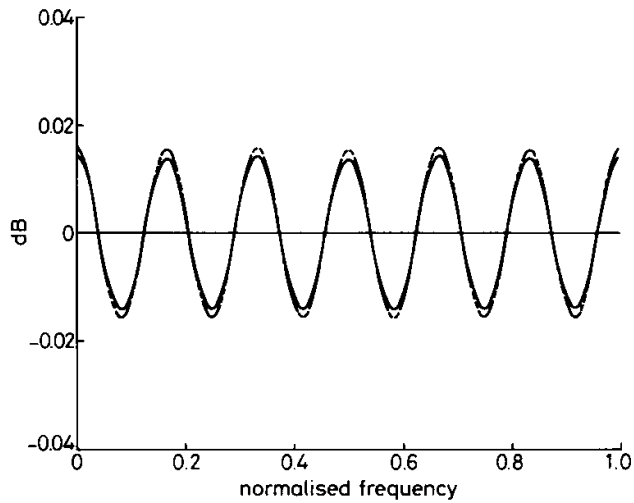

Fig. 4 Reconstruction error for the 1-D case

Table 1: Comparison of computational complexity for the 1-D case

\begin{tabular}{lll} 
& Real additions & Real multiplications \\
\hline Proposed design method & 360 & 800 \\
Numerical design method & $1.3 \times 10^{4}$ & $1.5 \times 10^{4}$ \\
\hline
\end{tabular}

atively search the filter coefficients $g(0), g(1), \ldots, g(15)$ The number of iterations was 200 . For comparison, the direct numerical optimisation of eqn. 5 based on a steepest-descent algorithm was also performed. The FFT size used was 128 . Fig. 3 shows the frequency response of $G(\omega)$ using both methods. The corresponding reconstruction errors are shown in Fig. 4. Table 1 compares the computational complexities in terms of the number of operations required at each iteration. Table 2 lists the

Table 2: Coefficients of designed 1-D prototype filter

\begin{tabular}{llc}
\hline & Proposed design method & Numerical design method \\
\hline$g_{0}=g_{15}$ & -0.002699048 & $0.733213933 \times 10^{-2}$ \\
$g_{1}=g_{14}$ & -0.009523474 & $-0.105630515 \times 10^{-1}$ \\
$g_{2}=g_{13}$ & -0.022380432 & $-0.242815156 \times 10^{-1}$ \\
$g_{3}=g_{12}$ & -0.003564694 & $-0.418084883 \times 10^{-2}$ \\
$g_{4}=g_{11}$ & 0.067013556 & $0.662609824 \times 10^{-1}$ \\
$g_{5}=g_{10}$ & 0.175784464 & 0.176067248 \\
$g_{6}=g_{9}$ & 0.288924903 & 0.289141969 \\
$g_{7}=g_{8}$ & 0.361273963 & 0.360878687 \\
\hline
\end{tabular}

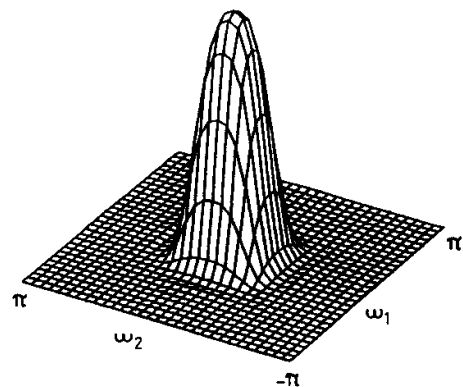

Fig. 5 Magnitude response of $G\left(\omega_{1}, \omega_{2}\right)$ for nonseparable case

Table 3: Comparison of computational complexity for the 2-D case

\begin{tabular}{lll}
\hline & Real additions & Real multiplications \\
\hline Proposed design method & $8.4 \times 10^{4}$ & $2.0 \times 10^{5}$ \\
Numerical design method & $3.2 \times 10^{8}$ & $3.7 \times 10^{8}$ \\
\hline
\end{tabular}

computed filter coefficients for this case. From these results, we observe that the proposed analytical design method is superior to the direct numerical optimisation algorithm.

\subsection{2-D nonseparable design example}

In this case, the 2-D FIR lowpass prototype filter is nonseparable with $M_{1}=M_{2}=16$. Both $N_{1}$ and $N_{2}$ were set to three and the relative weight $\alpha$ to 1000 . Fig. 5 shows the frequency response of $G\left(\omega_{1}, \omega_{2}\right)$ using the proposed 2-D design method described in Section 3. Table 3 compares the computational complexities for the 2-D case. Owing to the huge computational burden shown in Table 3 when using the numerical method, only the 2-D filter design was performed. Table 4 lists the designed filter coefficients. To demonstrate the reconstruction capability of the resulting 2-D PFB, we used a $128 \times 128$

\begin{tabular}{|c|c|c|c|c|c|c|c|c|}
\hline$g(m, n)$ & $m=0$ & 1 & 2 & 3 & 4 & 5 & 6 & 7 \\
\hline $\begin{array}{r}n=0 \\
1 \\
2 \\
3 \\
4 \\
4 \\
5 \\
6 \\
7\end{array}$ & $\begin{array}{r}0.00017 \\
0.00033 \\
0.00028 \\
-0.00019 \\
-0.00118 \\
-0.00253 \\
-0.00386 \\
-0.00469\end{array}$ & $\begin{array}{r}0.00033 \\
0.00062 \\
0.00054 \\
-0.00033 \\
-0.00218 \\
-0.00472 \\
-0.00720 \\
-0.00875\end{array}$ & $\begin{array}{r}0.00028 \\
0.00054 \\
0.00047 \\
-0.00028 \\
-0.00189 \\
-0.00410 \\
-0.00627 \\
-0.00761\end{array}$ & $\begin{array}{r}-0.00019 \\
-0.00033 \\
-0.00028 \\
0.00020 \\
0.00119 \\
0.00253 \\
0.00384 \\
0.00465\end{array}$ & $\begin{array}{r}-0.00118 \\
-0.00218 \\
-0.00189 \\
0.00119 \\
0.00765 \\
0.01650 \\
0.02516 \\
0.03053\end{array}$ & $\begin{array}{r}-0.00253 \\
-0.00472 \\
-0.00410 \\
0.00253 \\
0.01650 \\
0.03565 \\
0.05438 \\
0.06601\end{array}$ & $\begin{array}{r}-0.00386 \\
-0.00720 \\
-0.00627 \\
0.00384 \\
0.02516 \\
0.05438 \\
0.08297 \\
0.10072\end{array}$ & $\begin{array}{r}-0.00469 \\
-0.00875 \\
-0.00761 \\
0.00465 \\
0.03053 \\
0.06601 \\
0.10072 \\
0.12228\end{array}$ \\
\hline
\end{tabular}


2-D image as the input signal. Fig. 6 shows the simulation results. The difference image shown in Fig. $6 c$ is equal to $\mid(x(m, n)-\hat{x}(m, n) \mid$, where $x(m, n)$ denotes the original image and $\hat{x}(m, n)$ the reconstructed image. For the displayed images, the darkest image pixel has gray value 0 , while the brightest image pixel has gray value
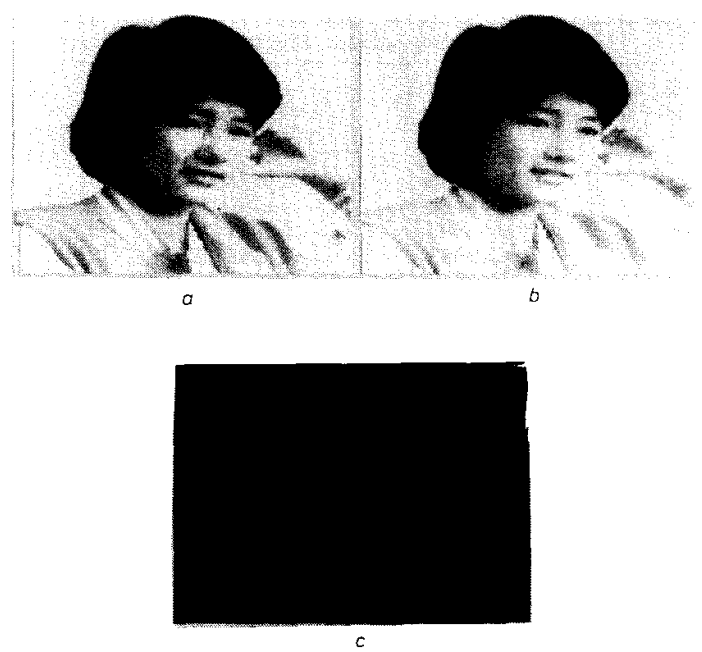

Fig. 6 Simulation results for the 2-D nonseparable case a Original image

$b$ Reconstructed image

c. Difference image

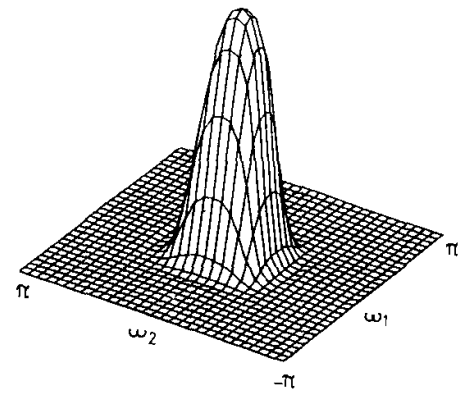

Fig. 7 Magnitude response of $G\left(\omega_{1}, \omega_{2}\right)$ for separable case

255. From Fig. 6, we observe that the reconstructed image is almost the same as the original image.

\subsection{2-D separable design example}

Here we consider the same design example treated above, except that the 2-D prototype filter is separable. Fig. 7 shows the frequency response of $G\left(\omega_{1}, \omega_{2}\right)$ designed by using the proposed 1-D design method described in
Section 2. Table 5 lists the designed filter coefficients. Fig. 8 shows the simulation results. Again, the difference image is almost invisible.

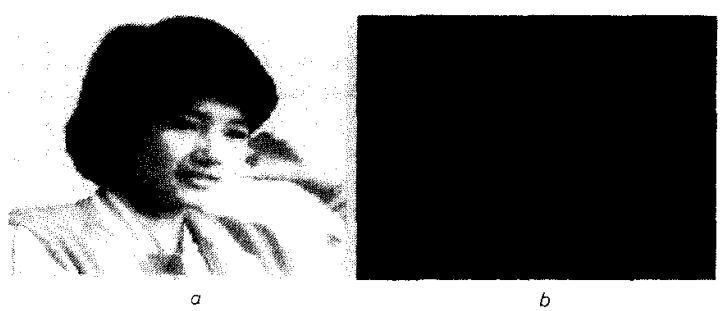

Fig. 8 Simulation results for the 2-D separable case a Reconstructed image $b$ Difference image

\section{Conclusions}

In this paper, we have presented an analytical method for the design of the lowpass prototype filter for a newlydeveloped polyphase filter bank. A closed analytical formula has been derived to represent an appropriately defined approximation error measure in the design process. Based on this formula, computing the filter coefficients by minimising the approximation error can be performed utilising a simple direct search algorithm. This leads to a reduction in the complexity of operations in the filter design process. The 1-D theoretical results have also been extended to two dimensions. An analytical formula for designing the new 2-D polyphase filter bank with a 2-D nonseparable prototype filter is derived. This formula facilitates the design work in the 2-D case. If the 2-D polyphase filter bank is constructed using a 2-D separable prototype filter, the developed 1-D analytical formula can be employed to further reduce the design complexity. The theoretical work has been confirmed by computer simulations.

6

\section{References}

1 ESTEBAN, D., and GALAND, C.: 'Application of quadrature mirror filter to split-band voice coding schemes'. Proc. IEEE Int. Conf. Acoust., Speech, Signal Processing, Hartford, CT, USA, May 1977, pp. 191-195

2 CROCHIERE, R.E.: 'Digital signal processor: sub-band coding' Bell Syst. Tech. J. 1981, 60, pp. 1633-1653 3 BELLANGER, M.G., and DAGUET, J.L.: 'TDM-FDM trans-
multiplexer: digital polyphase and FFT', IEEE Trans. Commun., 1974, COM-22, pp. 1199-1204

4 VETTERLI, M.: 'Perfect transmultiplexers'. Proc. IEEE Int. Conf. Acoust., Speech, Signal Processing, Tokyo, Japan, April 1986, pp. $2567-2570$

5 VARY, P., and HEUTE, U.: 'A short-time spectrum analyzer with polyphase network and DFT', Signal process., 1980, 2, pp. 55-65

6 HEUTE, U., and VARY, P.: A digital filter bank with polyphase HEUTE, U., and VARY, P.: 'A digital filter bank with polyphase
network and FFT hardware: measurements and applications', network and FFT hardware: meas

Signal Process., 1981, 3, pp. 307-319
7 VETTERLI, M.: 'Multidimensional sub-band coding: some theory and algorithms', Signal Process., 1984, 6, pp. 97-112

Table 5: Coefficients of the designed 2-D separable prototype filter

\begin{tabular}{|c|c|c|c|c|c|c|c|c|}
\hline$g(m, n)$ & $m=0$ & 1 & 2 & 3 & 4 & 5 & 6 & 7 \\
\hline $\begin{array}{r}=0 \\
1 \\
2 \\
3 \\
4 \\
5 \\
5 \\
6 \\
7\end{array}$ & $\begin{array}{r}0.00018 \\
0.00033 \\
0.00029 \\
-0.00018 \\
-0.00117 \\
-0.00252 \\
-0.00385 \\
-0.00467\end{array}$ & $\begin{array}{r}0.00033 \\
0.00062 \\
0.00054 \\
-0.00033 \\
-0.00218 \\
-0.00471 \\
-0.00719 \\
-0.00873\end{array}$ & $\begin{array}{r}0.00029 \\
0.00054 \\
0.00047 \\
-0.00029 \\
-0.00190 \\
-0.00411 \\
-0.00627 \\
-0.00761\end{array}$ & $\begin{array}{r}-0.00018 \\
-0.00033 \\
-0.00029 \\
0.00018 \\
0.00116 \\
0.00250 \\
0.00382 \\
0.00464\end{array}$ & $\begin{array}{r}-0.00117 \\
-0.00218 \\
-0.00190 \\
0.00116 \\
0.00762 \\
0.01647 \\
0.02514 \\
0.03053\end{array}$ & $\begin{array}{r}-0.00252 \\
-0.00471 \\
-0.00411 \\
0.00250 \\
0.01647 \\
0.03562 \\
0.05437 \\
0.06600\end{array}$ & $\begin{array}{r}-0.00385 \\
-0.00719 \\
-0.00627 \\
0.00382 \\
0.02514 \\
0.05437 \\
0.08279 \\
0.10074\end{array}$ & $\begin{array}{r}-0.00467 \\
-0.00873 \\
-0.00761 \\
0.00464 \\
0.03053 \\
0.06600 \\
0.10074 \\
0.12230\end{array}$ \\
\hline
\end{tabular}


8 WOODS, J.W., and O'NEIL, S.D.: 'Subband coding of images', IEEE Trans. Acoust., Speech, Signal Process., 1986, ASSP-34, pp. 1278-1288

9 WACKERSREUTHER, G.: 'On two-dimensional polyphase filter banks', IEEE Trans. Acoust., Speech, Signal Process., 1986, ASSP34, pp. 192-199

10 JOHNSTON, J.D.: 'A filter family designed for use in quadrature mirror filter banks'. Proc. IEEE Int. Conf. Acoust., Speech, Signal Processing, Denver, CO, USA, 1980, pp. 291-294

11 JAIN, V.K., and CROCHIERE, R.E.: 'Quadrature mirror filter design in time domain', IEEE Trans. Acoust., Speech, Signal Process., 1984, ASSP-32, pp. 353-361

12 MINTZER, F.: 'Filters for distortion-free two-band multirate filter banks', IEEE Trans. Acoust., Speech, Signal Process., 1985, ASSP33, pp. $626-630$

13 CHU, P.: 'Quadrature mirror filter design for an arbitrary number of equal bandwidth channels', IEEE Trans. Acoust. Speech, Signal Process., 1985, ASSP-33, pp. 203-218

14 VAIDYANATHAN, P.P. 'Theory and design of M-channel maximally decimated quadrature mirror filters with arbitrary $M$, having perfect reconstruction property', IEEE Trans. Acoust., Speech, Signal Process., 1987, ASSP-35, pp. 476-492

15 DOGANATA, Z., VAIDYANATHAN, P.P., and NGUYEN, T.Q. 'General synthesis procedure for FIR lossless transfer matrices, fo perfect-reconstruction multirate filter bank applications', IEEE Trans. Acoust., Speech, Signal Process., 1988, ASSP-36, pp. 15611574

16 WACKERSREUTHER, G.: 'A novel approach to the design of filters for filter banks'. Proc. IEEE Int. Conf. Acoust., Speech, Signal Processing, Tampa, FL, USA, 1985, pp. 2.9.1-2.9.4

17 WACKERSREUTHER, G.: 'Some new aspects of filters for filter banks', IEEE Trans. Acoust., Speech, Signal Process., 1986, ASSP34, pp. 1182-1200

I8 PIRANI, G., and ZINGARELLI, $V$. "An analytical formula for the design of quadrature mirror filters', IEEE Trans. Acoust., Speech, Signal Process., 1984, ASSP-32, pp. 645-648

19 VISCITO, E., and ALLEBACH, J.P.: 'Design of perfect reconstruction multi-dimensional filter banks using cascaded Smith-form matrices'. Proc. IEEE Int. Symp. Circuits Syst., Helsinki, Finland, 1988, pp. 831-834

20 LEE, J.-H and YOUNG, T-T.: 'New polyphase filter bank-based analysis/synthesis systems', IEEE Trans. Acoust., Speech, Signal Process., 1990, ASSP-38, pp. 876-880

21 LEE, J-H., and YOUNG, T-T. 'Two-dimensional polyphase filter banks with arbitrary number of subband channels'. Proc. IEEE Int Symp. Circuits Syst., Helsinki, Finland, 1988, pp. 1265-1268 